\title{
EAVN observations along with EHT for M87 in 2017
}

\author{
Yuzhu Cui*, Kazuhiro Hada, Mareki Honma \\ Mizusawa VLBI Observatory, National Astronomical Observatory of Japan, SOKENDAI \\ E-mail: yuzhu.cui@nao.ac.jp, kazuhiro.hada@nao.ac.jp
}

\section{Motoki Kino}

National Astronomical Observatory of Japan

\section{Hyunwook Ro}

Department of Astronomy, Yonsei University

Korea Astronomy and Space Science Institute (KASI)

\section{Jongho Park}

Department of Physics and Astronomy, Seoul National University

\section{Masanori Nakamura}

Institute of Astronomy \& Astrophysics, Academia Sinica (ASIAA)

\section{on behalf of the EAVN AGN Science Working Group}

\begin{abstract}
After the successful combination of VERA in Japan and KVN in Korea (KaVA), the East Asian VLBI Network (EAVN) is now expanding by including Chinese antennas, significantly enhancing the angular resolution and sensitivity. One of the key science goals of EAVN project is to better understand the formation of Active Galactic Nuclei (AGN) jets. In early 2017, we started an intensive EAVN monitoring on the nearby galaxy M87. This source offers a privileged opportunity to probe the jet launching and formation scales thanks to the proximity and large mass of the central black hole. In total we obtained 14 epochs of M87 from March to May 2017 where a number of stations in East Asia joined (so-called the "EAVN campaign 2017"). For the first time, we obtained M87 images with KaVA + Tianma at both 22 and $43 \mathrm{GHz}$. Moreover, the EAVN campaign 2017 was performed near-in-time to the EHT+ALMA observation of M87. Quasi-simultaneous EAVN observations at the lower frequencies may offer complementary information to properly interpret the EHT images. Here we report some preliminary results on these EAVN observations.
\end{abstract}

14th European VLBI Network Symposium \& Users Meeting (EVN 2018)

8-11 October 2018

Granada, Spain

\footnotetext{
* Speaker.
} 


\section{Introduction}

The relativistic jets in Active Galactic Nuclei (AGN) are the most energetic phenomena in the Universe, and believed to be powered by the accretion onto the central supermassive black holes (SMBH). To better understand the formation of AGN jets, the key is to directly resolve the innermost jet regions close to the central black hole. The high-resolution VLBI observations that achieve milliarcsecond scale are the unique way to do that. To this end, we selected the nearby radio galaxy M87 $\left(D=16.7 M p c, 1\right.$ mas $\left.\approx 0.081 p c, M_{\mathrm{BH}} \sim(3.3-6.2) \times 10^{9} M_{\odot}\right)$ as our target in the first monitoring program with the East Asian VLBI Network (EAVN) which were performed from January to May 2017 (EAVN campaign 2017). M87 is the one of the most famous AGNs with relativistic jets, and thanks to its proximity, we can directly access the predicted scales of jet collimation and acceleration very close to SMBH with VLBI [1,2]. According to the standard MHD models, AGN jets are driven by the magnetic field and accelerated by the conversion from magnetic to kinetic energy. To better understand these acceleration scales, it is the key to investigate the kinetic and spectral information based on the multi-epoch and multi-frequency VLBI observations.
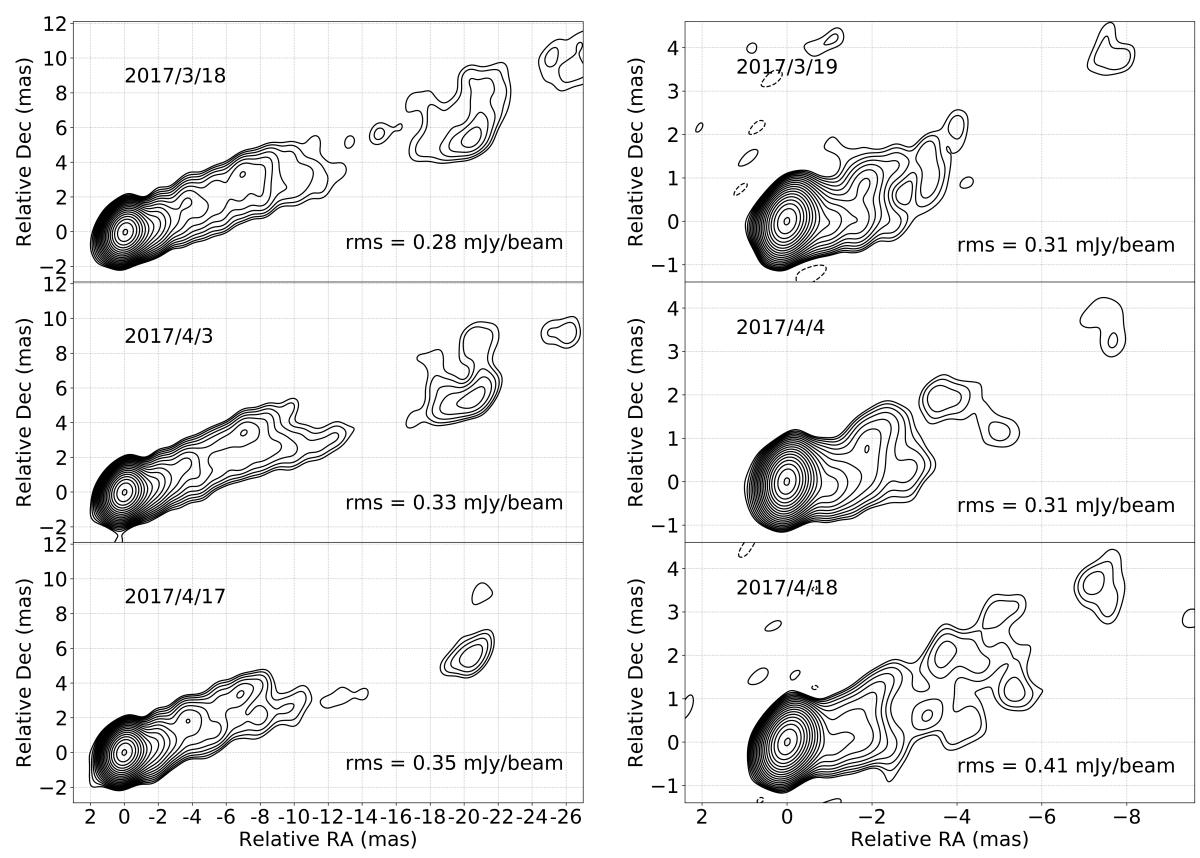

Figure 1: Six representative structure images of M87 at 22 (left) and $43 \mathrm{GHz}$ (right) obtained from EAVN campaign 2017. A common beam size of 1.56 mas $\times 0.96$ mas,$-12^{\circ}$ or 0.68 mas $\times 0.57$ mas, $0.24^{\circ}$ is used for $22 / 43 \mathrm{GHz}$, respectively. Contours start from $1.9 \mathrm{mJy} /$ beam. Off-source rms image noise levels are shown.

The EAVN is an international VLBI collaboration in East Asia, currently among Japan, Korea and China. The KVN and VERA Array (KaVA) [3], as a precursor of the EAVN, has biweekly conducted a large KaVA-M 87 monitoring program at $22 \mathrm{GHz}$ since 2013 [4]. Concurrently, further efforts have been made to expand the network by including Tianma and Urumuqi as well as other East Asian stations [5]. To better trace the M87 activities with higher angular resolution and sensitivity, from early 2017, we started dense monitoring of M87 with EAVN at 22/43 GHz. The 
angular resolution is up to 0.55 mas ( 0.63 mas) at $22(43) \mathrm{GHz}$, respectively. Fourteen epochs were obtained in total. For each epoch, a continuous 5-7-hour track was conducted. The observing time interval is about 4-8 days which is the shortest one by now. This intensive sampling may significantly reduce mis-classification of moving components and also provide a chance to detect fast motion that is difficult to find with the low cadence monitoring. Moreover, M87 was for the first time observed by EHT+ALMA at $230 \mathrm{GHZ}$ in April 2017. This may allow the first imaging of the black hole shadow and jet-launching regions at scales of a few Schwarzschild radii. However, due to the sparse uv-coverage of the EHT, a proper interpretation of the EHT images may require contemporaneous complementary observations at the lower frequencies. It also makes EAVN campaign 2017 distinctly unique. In the following sections, we will introduce some preliminary results obtained from EAVN data.

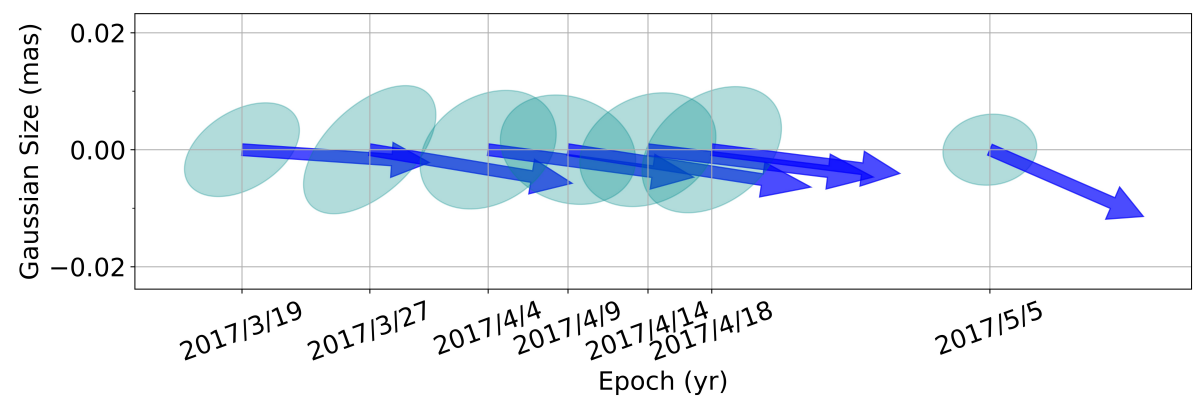

Figure 2: The elliptical Gaussian component of the core at $43 \mathrm{GHz}$ obtained from EAVN and VLBA observations. The last one on May 5th is obtained from a VLBA data. The length and direction of blue arrows indicate the distance and direction of the second circular Gaussian component with respect to the core.

\section{Evolution of structure}

The images in Figure 1 are picked from EAVN campaign 2017 observed by KaVA+Tianma. It shows the overall jet appearance at $22 / 43 \mathrm{GHz}$ with natural weighting. The dynamic range of the images reaches to 4600 . At $22 \mathrm{GHz}$, we can clearly trace the jet down to 21 mas from the core thanks to the dramatic improvement of imaging capability compared with only KaVA. From the $43 \mathrm{GHz}$ images, we can sufficiently resolve the innermost jet region. The jet direction within 1-2 mas from the core is largely horizontal with the southern limb being brighter. Beyond 2 mas from the core, the jet direction becomes along with a position angle of $-72^{\circ}$ which is well known for the large-scale jet. The horizontal position angle can be clearly checked in Figure 2. This is consistent with the transverse motion reported by Walker et al. 2018 [2] who suggest an 8-10 yr quasi-periodicity based on 17 years of the VLBA data. We followed the same measurement and confirmed the agreement of sideways shift at 2 mas based on our EAVN data. It might be related to a helical flow although more investigations are needed. As for the possible connection to EHT, if we assume that the innermost jet direction is constant from the EAVN scale down to EHT scale, the initial jet launching might be seen in the direction toward the west in the horizon-scale EHT image. Regarding the kinematics of the jet, our preliminary analysis suggests the presence of some fast motion $\left(\beta^{a p p} \equiv v^{a p p} / c>2\right)$ near the jet base. More detailed measurements are in progress. 

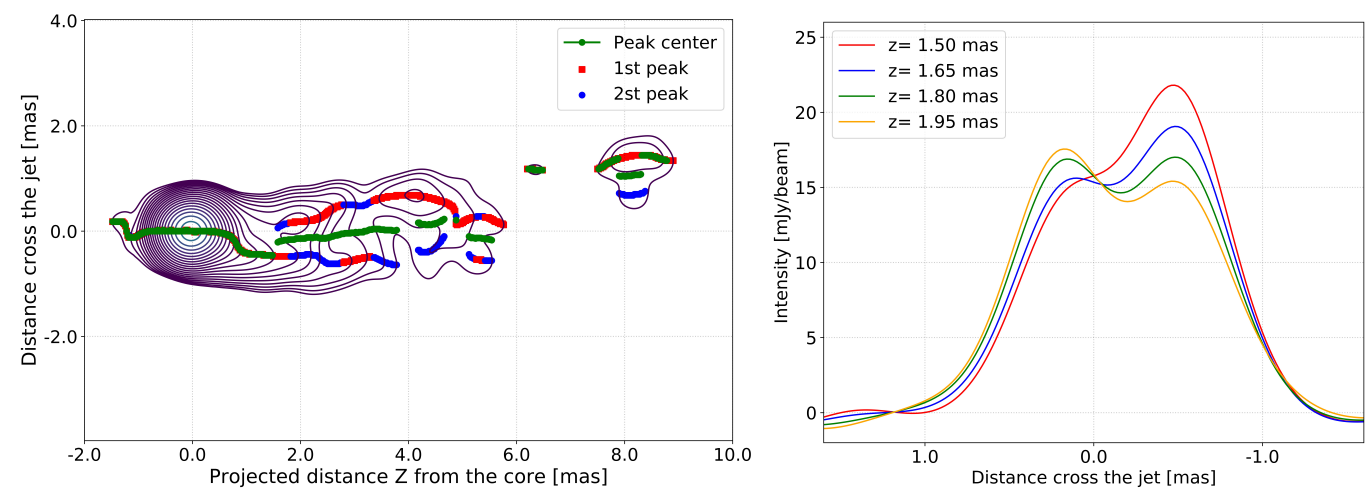

Figure 3: Left: stacked M 87 image at $Q$ band after rotating on the sky by $-18^{\circ}$ in order to align the jet central axis same with previous work [6] to horizontal direction. So the positive y-axis part represents the northern side of the jet. Each epoch image is restored with the 0.6 mas circular Gaussian beam which is equivalent to the angular resolution of EAVN at Q band. Contour levels are $(1,1,1.4,2,2.8, \ldots) \times 0.47$ mJybeam $^{-1}$. The transverse profile is obtained by the slicings perpendicular to the jet axis. The red and blue points presents the first and second highest peak, respectively. The center of the peak(s) is in green color. Right: intensity profiles cross the jet at 1.50,1.65,1.80 and 1.95 mas.

\section{Limb-brightening profile}

To investigate the transverse profile, we firstly check the time-averaged emission after stacking EAVN image at $\mathrm{Q}$ band (Figure 3). The individual data is restored with a circular 0.6 mas Gaussian beam. After aligning the restored images with the peak positions, we adopt the jet axis $\mathrm{PA}=-72^{\circ}$ as previous work $[2,6]$ to divide the jet into northern and southern sides. The slicing perpendicular to the jet axis shows clear limb-brightening profile at core separation larger than 1.5 mas. The first and second brightest peaks change their positions on the southern and northern ridges by turn along the jet which can be directly seen in Figure 3. It may be related to the jet pattern. The intensity distribution at the innermost region $(<1.5$ mas $)$ shows only one Gaussian component on the southern side due to the large restored beam size. Within this region, the peak center overlaps with the first brightest peak position and the southern side is much brighter than the northern side. We also checked the image with slightly smaller beam $\left(0.4 \mathrm{mas} \times 0.2 \mathrm{mas}, 1^{\circ}\right)$ to check the innermost jet region. Jet also shows clear two ridges within 1.5 mas from the core. More precise measurement of jet position angle, jet width, collimation profile and transverse motion is under investigation.

\section{Spectral index Property}

The spectral index distribution of AGN jets obtained from the multi-epoch and multi-frequency observations tells us some key physical properties of the sources, such as the particle energetics and the magnetic field. By correcting the core-shift effect with 2D image cross-correlation technique [7], we got spectral index maps of the M87 jet with the 3 pairs of contemporaneous EAVN $22 / 43 \mathrm{GHz}$ observations (see Figure 1). In Figure 4 we show a spectral index map that was averaged over these 3 pairs. We define the spectral index $\alpha$ by the relation $S_{v} \propto v^{\alpha}$ where $S_{v}$ is the flux density at a frequency $v$. From the stacked spectral index map we can get the average value 


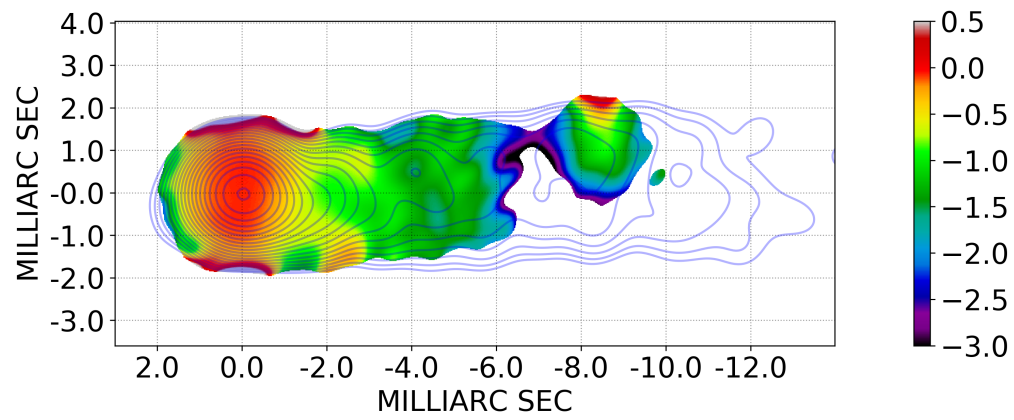

Figure 4: Spectral-index map of the stacked image including 3 pairs of quasi-simultaneous epochs at 22 and $43 \mathrm{GHz}$ (namely the data shown in Figure 1). A common beam size of $22 \mathrm{GHz}(1.2 \mathrm{mas} \times 1.2 \mathrm{mas})$ is used for both frequencies. The core shift was measured by doing a 2D cross-correlation image alignment which is around $20 \mu$ as along the jet direction ( $\mathrm{PA}=-72^{\circ}$ with respect to north). Core shift has been applied in this figure.

of spectral index is $\alpha \sim-1.0$ for the optically thin region which is further than 2 mas from the core. The combination of spectral and kinetic information will be useful to constrain the magnetic field. In addition, the spectral information could also limit the expected integrated flux density of the emission at $230 \mathrm{GHz}$. The EAVN data are being analyzed in more detail to make a conclusive summary of M87 jet activities in 2017 and provide more information to better interpret EHT images.

\section{References}

[1] K. Hada, et al. High-sensitivity $86 \mathrm{GHz}(3.5 \mathrm{~mm})$ VLBI Observations of M87: Deep Imaging of the Jet Base at a Resolution of 10 Schwarzschild Radii, ApJ 817, 131 (2016)

[2] R. C. Walker, et al. The Structure and Dynamics of the Subparsec Jet in M87 Based on 50 VLBA Observations over 17 Years at $43 \mathrm{GHz}$, ApJ 855, 128 (2018)

[3] K. Niinuma, et al. VLBI observations of bright AGN jets with the KVN and VERA Array (KaVA): Evaluation of imaging capability, PASJ 66, 103 (2014)

[4] K. Hada, et al. Pilot KaVA monitoring on the M 87 jet: Confirming the inner jet structure and superluminal motions at sub-pc scales, PASJ 69, 71 (2017)

[5] K. Wajima, et al. The East-Asian VLBI Network, ASP Conf. Ser. 502, Frontiers in Radio Astronomy and FAST Early Sciences Symposium 2015, 81 (2016)

[6] K. Hada, et al. An origin of the radio jet in M87 at the location of the central black hole, Nature 477, $185(2011)$

[7] S.M. Croke, and D.C. Gabuzda Aligning VLBI images of active galactic nuclei at different frequencies, MNRAS 386, 619 (2008) 